recurrence patients had mean age $=42$ yrs (range 18-83 yrs) and 6 were males. The sites of relapse were: pulmonary in 6 cases, 3 patients had intracranial tuberculomas, 2 patients had bony TB, 3 patients had TB lymphadenitis. The mean time to relapse/recurrence was 41 months (range 2-96 mo). All 14 patients responded favourably to re-treatment.

The true relapse rate of TB treated at the centre was $0.4 \%$. The age, gender and ethnicity of the relapse cases were similar to the overall TB case-mix.

COnclusions Our true relapse/recurrence rate of TB is very low, and had no obvious risk factors. We cannot determine retrospectively whether these were recurrence or reinfection, but strain typing (DNA fingerprinting) could differentiate these.

\section{M42 INCREASING COMPLEXITY OF TREATING TB IN OLDER PATIENTS}

J Barrett, GA O'Hara, A Nundoll, N Price, H Milburn, RAM Breen. Guys and St Thomas; NHS Foundation Trust, London, UK

\subsection{6/thoraxinl-2014-206260.430}

Introduction Older adults remain an important reservoir of tuberculosis (TB) infection in the UK. Waning cellular immune responses, more frequent co-morbidities such as diabetes and malignancy, and increased polypharmacy may all modulate clinical presentation, treatment tolerability and ultimately outcomes when compared to younger individuals with TB. We sought to investigate this in our population.

Methods Retrospective study of all adults over 60 diagnosed with TB during a five year period at one hospital trust. Case-note and electronic record review established baseline disease features, comorbidities, pre-morbid immune suppression including HIV status, TB-related outcomes and death. A randomly selected control group of identical size, containing adults aged 16-59 who were treated for TB during the same period, was used for comparison.

Results Forty-eight cases aged $>60$ years at $\mathrm{TB}$ diagnosis were identified. The case and control groups are described in the Table. Multi-lobar pulmonary disease was significantly more common in the $>60$ year old, as was diabetes, other significant co-morbidities and non-HIV immune suppression. Whilst treatment regimen discontinuation or alteration was more common in the $>60$ year old group (7 (14\%) versus $3(6 \%)$ if 60 years old but none $(0 \%)$ of the younger group; whilst deaths after completion of TB treatment have been observed in $8(17 \%)$ and $1(2 \%)$ of cases respectively (no post-treatment death was related to $\mathrm{TB}$ in either group).

\begin{tabular}{llll}
\hline Abstract M42 Table 1 & & \\
\hline & $\begin{array}{l}<0 \text { years old } \\
(\mathbf{n}=\mathbf{4 8})\end{array}$ & $\begin{array}{l}>60 \text { years old } \\
(\mathbf{n}=48)\end{array}$ & \\
\hline Mean age at diagnosis (range) & $34(16-57$ years) & $71(60-88$ years) & \\
Male & $24(54 \%)$ & $33(69 \%)$ & \\
Pulmonary disease & $34(70 \%)$ & $33(69 \%)$ & \\
- multi-lobar & $9(26 \%)$ & $17(52 \%)$ & $\mathrm{p}=0.046$ \\
Culture-confirmed TB & $38(79 \%)$ & $36(75 \%)$ & \\
HIV-infected & $6(13 \%)$ & 0 & $\mathrm{p}=0.026$ \\
Diabetes & 0 & $13(27 \%)$ & $\mathrm{p}<0.01$ \\
Significant immune suppression & $8(17 \%)$ & $29(60 \%)$ & $\mathrm{p}<0.01$ \\
Significant co-morbidities & $14(35 \%)$ & $36(75 \%)$ & $\mathrm{p}<0.01$ \\
\hline
\end{tabular}

Conclusions Our observations of older patients presenting with more extensive pulmonary disease, increased pre-diagnosis immune suppression and co-morbidities, as well as more frequent TB-related deaths and TB-regimen alterations, suggest that this group of TB patients are frequently in need of more intensive support during treatment than their younger counterparts. However if treatment can be tolerated and completed than TB outcomes do not appear to be affected by age.

\section{M43 THE ACCURACY OF CLINICAL TB DIAGNOSES IN CULTURE NEGATIVE PATIENTS}

L Maynard Smith, NP Jayasekera, VM Macavei, TC O'Shaughnessy. Newham University Hospital, London, UK

\subsection{6/thoraxjnl-2014-206260.431}

Background NICE guidelines for TB diagnosis recommend that sputum is obtained for culture for all suspected cases of pulmonary $\mathrm{TB}$, and biopsies for all cases extrapulmonary TB. As results can take 6 weeks, treatment initiation decisions are frequently made without microbiological confirmation.

Aim This study set out to examine the accuracy of clinical diagnoses in a high incidence area, and the basis for these decisions. Methods The data entered onto the national TB database was used to obtain a list of patients for whom no culture results had been recorded. Clinic letters, laboratory records and imaging were examined to determine whether samples had been sent for culture, how diagnoses were made in the event of negative results, and if alternative diagnoses were concluded.

Results Of 323 patients on the database, $7 \%$ had no samples sent for culture. There were 109 culture negative patients, of whom 13 (4\% all cases) had alternative diagnoses. A combination of relevant history and imaging was the most commonly used method of diagnosis when culture was negative (47\%). Histology was used in $17 \%$ patients and Mantoux or IGRA testing supported initiating treatment in $39 \%$ cases. The database was missing positive culture results for 102 patients, of which four were MDR TB.

Conclusions In this study, we found accurate initial clinical diagnoses, with only $4 \%$ patients subsequently obtaining alternative diagnoses. Most diagnoses were made on the basis of relevant history and imaging. Of concern are the $7 \%$ patients for whom tissue was never sent for culture. This is likely to be an underestimate when including all patients initially suspected of TB, raising the possibility of missed diagnoses. The utility of Mantoux and IGRA testing in active disease is now disputed. It is hoped with inter-specialty education regarding the importance of culture and futility of immunological based assays, the proportion of patients with suspected TB who have sputum or tissue sent for culture increases. Accurate recording of MDR-TB on the national TB database needs to be improved, to enable efficient monitoring of intervention programmes.

\section{COPD: co-morbidities, deficiencies and interventions}

\section{M137 CAN STEROID INSENSITIVITY IN COPD PATIENTS BE RESTORED USING VITAMIN D?}

D Mukherjee, D Parekh, R Dancer, M Ungurs, H Khiroya, AM Turner. Queen Elizabeth Hospital Research Laboratories, University of Birmingham, Birmingham, UK

10.1136/thoraxjnl-2014-206260.432 
Introduction In many chronic diseases vitamin $\mathrm{D}$ has been proposed as an adjunctive anti-inflammatory therapy. Vitamin D upregulates MKP1, thereby downregulating p38 phosphorylation and the NFKB inflammatory cascade (Zhang et al, J Immunol. 2012;188(5):2127-35). Steroids exert anti-inflammatory effects via this cascade, and exhibit synergy with vitamin $\mathrm{D}$ for some effects (Yu et al, Journal of the National Cancer Institute. 1998;90(2):134-41). Patients with COPD have chronic pulmonary inflammation, with upregulation of NFKB, yet do not exhibit a good response to steroids. Vitamin D therapy has been trialled in COPD patients, albeit with disappointing results (Lehouck et al, Annals of internal medicine. 2012;156(2):10514). We hypothesised that COPD patients' inflammatory response would differ from health, and that vitamin D would exhibit synergy with steroids in vitro to improve this.

Methods PBMCs isolated from 10 COPD patients and 10 healthy control subjects were incubated with LPS, vitamin D, dexamethasone, a p38 MAPK inhibitor or combinations of these agents. Supernatants were harvested for TNF and IL6 measurements (ELISA).

Results LPS caused a marked rise in IL6 in both healthy controls $(p=0.044)$ and COPD patients $(p=0.008)$. IL6 reduction with vitamin $\mathrm{D}$ was only seen in health. IL6 reduction with addition of dexamethasone was not statistically significant $(\mathrm{p}=$ 0.636) in COPD. Combinations of agents failed to produce any additional benefit in both health and COPD.

The response to vitamin $\mathrm{D}$ was heterogeneous; half of healthy subjects showed an anti-inflammatory response but in COPD only $12.5 \%$ of patients exhibited this. The difference in response rate was not significant $(\mathrm{p}=0.120$, Fishers exact test), though this may be due to low power. Similarly reduced response rate to dexamethasone was seen in COPD.

Conclusion Vitamin D does not enhance the anti-inflammatory effect of steroids. The anti-inflammatory effects of vitamin D are no different between COPD and health; variability of response may be one reason for lack of effect of vitamin D in clinical trials to date in COPD patients.

\section{M138 DO STANDARD CARDIOVASCULAR RISK SCORES IDENTIFY RISK IN PATIENTS WITH COPD?}

ME John, S Hussain, M Al Haddad, CE Bolton. University of Nottingham, Nottingham, UK

\subsection{6/thoraxjnl-2014-206260.433}

Introduction Patients with COPD have increased risk of cardiovascular (CV) disease compared to smokers without COPD, ${ }^{1}$ with over $25 \%$ of deaths CV related. $^{2}$ Several CV risk calculators for the general population exist but it is unclear whether they are applicable for COPD.

Hypothesis

Standard CV risk calculators do not identify the increased risk in patients with COPD.

Methods Subjects with a smoking history $>10$ pack years, with and without COPD, were assessed at clinical stability, COPD n $=191$ and controls $\mathrm{n}=106$. Post-bronchodilator spirometry and blood pressure were performed, blood taken for lipids and self-reported medical and smoking history recorded. In those without documented established CV disease or diabetes (COPD $\mathrm{n}=135$ and controls $\mathrm{n}=88$ ), 10 year CV risk was calculated using ACC/ $\mathrm{AHA}^{3}$ and NHLBI[4] calculators.

Results Both groups were well matched for gender and mean arterial blood pressure (MAP), with the COPD group slightly older, Table 1. Mean CV risk scores were similar between

\begin{tabular}{lll} 
Abstract M138 Table $\mathbf{1}$ & \\
\hline $\begin{array}{l}\text { Mean (SD) } \\
\text { unless stated otherwise }\end{array}$ & COPD & Controls \\
\hline $\mathrm{n}$ & 135 & 88 \\
Age (years) & $67(8)$ & $65(10)$ \\
Gender male $\mathrm{n}(\%)$ & $79(59)$ & $56(64)$ \\
FEV $\%$ predicted & $56(19)$ & $100(14)$ \\
Smoking pack years & $45(26)$ & $29(19)$ \\
Smoking status current: ex $\mathrm{n}(\%)$ & $40(30): 95(70)$ & $18(20): 70(80)$ \\
MAP (mmHg) & $105(13)$ & $105(12)$ \\
ACC/ AHA risk score & $19(12)$ & $16(12)$ \\
NHLBI risk score & $11(8)$ & $10(8)$ \\
\hline
\end{tabular}

patients with COPD and controls, Table 1, ACC/AHA p $=0.16$ and NHLBI $\mathrm{p}=0.59$. When using an established cut-off point of $20 \%$ for high 10 year CV risk, similar proportions were identified as high risk: the ACC/AHA calculator - $37 \%$ of the patients with COPD and 33\% of controls; and with the NHLBI calculator $15 \%$ of the patients with COPD and $10 \%$ of controls were identified as high-risk.

Discussion Although nearly double the proportion of patients with COPD compared to controls with a smoking history have current $\mathrm{CV}$ disease or diabetes in this cross-sectional study, the increased risk of future incident $\mathrm{CV}$ disease in patients with COPD was not identified using standard calculators.

Supported by a NIHR BRF Fellowship.

\section{REFERENCES}

1 Feary, JR, et al. Thorax, 2010;65(11): p. 956-62

2 McGarvey, LP, et al. Thorax, 2007;62(5): p. 411-5

3 ACC/AHA Available from: http://my.americanheart.org/professional/StatementsGuidelines/Prevention-Guidelines_UCM_457698_SubHomePage.jsp

4 NHLBI Available from: http://crdrisk.nhlbi.nih.gov/

\section{M139 FRAILTY AND PREMATURE CARDIOVASCULAR AGEING IN COPD}

AM Albarrati, NS Gale, S Enright, M Munnery, I Munnery, S Saikia, JR Cockcroft, DJ Shale. Cardiorespiratory Medicine, Wales Heart Institute, Cardiff University, Cardiff, UK

\subsection{6/thoraxjn|-2014-206260.434}

Background Presence of comorbidities in chronic obstructive pulmonary disease (COPD) parallels the accumulation of multiple system deficits associated with ageing and assessed as frailty. An important association of frailty in the elderly is increased cardiovascular disease, which is also a major cause of mortality in COPD. ${ }^{1}$ However, frailty has not been extensively studied in COPD. We hypothesised that frailty in COPD would be associated with biomarkers of greater systemic involvement including cardiovascular and indicating premature cardiovascular ageing.

Methods Frailty was determined as a Frailty Index (FI) using the 61-element comprehensive geriatric assessment questionnaire in 500 patients with stable COPD, confirmed with spirometry, and 150 non-COPD comparators. This cross-sectional study was taken from within the ARCADE study. Other assessments included body composition; handgrip strength (HGS); aortic pulse wave velocity (PWV); cardiac haemodynamics; 6 min walk distance (6MWD); Timed Up and Go (TUG) test; St George's Respiratory Questionnaire (SGRQ) and C-reactive protein (CRP). The FI was calculated by dividing the number of deficits that the patient had by the maximum, 61

Results Patients and comparators were similar for age, BMI and gender proportion. The FI was greater in the COPD group; 